\title{
Adnexectomy by poor man's transvaginal NOTES
}

\author{
Anneleen Reynders ${ }^{1}$ • Jan Baekelandt ${ }^{1}$
}

Received: 11 February 2015 / Accepted: 8 June 2015 /Published online: 18 June 2015

(C) Springer-Verlag Berlin Heidelberg 2015

\begin{abstract}
The purpose of this study was to demonstrate the feasibility and safety of adnexectomy by transvaginal natural orifice transluminal endoscopic surgery (vNOTES) for benign adnexal masses. Conventional, reusable laparoscopic instruments were used, inserted through aninexpensive, selfconstructed single port device. Between November 2013 and November 2014, 20 adnexectomies by vNOTES were performed by a single surgeon (BJ). Only conventional, reusable laparoscopic instruments were used. The self-constructed single port device was made by assembling a surgical glove, a wound protector, one reusable $10-\mathrm{mm}$ trocar, and four reusable 5-mm trocars. The adnexectomy was performed according to the technique for standard laparoscopic surgery, and the specimen was removed through the colpotomy incision. Patient and perioperative data were analysed. Twenty patients underwent adnexectomy by vNOTES, and no conversion to standard laparoscopy or laparotomy was necessary. Mean operation time was $32 \mathrm{~min}$ (20-50 $\mathrm{min}$ ); mean drop in hemoglobin level was $0.9 \mathrm{~g} / \mathrm{dl}(0-2.1 \mathrm{~g} / \mathrm{dl})$. There were no operative complications. Post-operative pain scores were very low. The mean size of the removed adnexal mass was $51.8 \mathrm{~mm}$ (35$110 \mathrm{~mm}$ ). Adnexectomy by vNOTES is feasible even for masses up to $110 \mathrm{~mm}$ and even when performed with reusable, conventional laparoscopic instruments. The main advantages of vNOTES are better cosmetics, low postoperative pain
\end{abstract}

Electronic supplementary material The online version of this article (doi:10.1007/s10397-015-0900-x) contains supplementary material, which is available to authorized users.

Jan Baekelandt

jan.baekelandt@imelda.be

1 Department of Obstetrics and Gynaecology, Imelda Hospital Bonheiden, Imeldalaan 9, 2820 Bonheiden, Belgium scores and easy removal of the specimen without spillage. This frugally innovative technique also enables surgeons to perform adnexectomies by vNOTES in low resource settings.

Keywords Minimally invasive surgery $\cdot$ Natural orifice endoscopic surgery $\cdot$ Transvaginal $\cdot$ Adnexectomy $\cdot$ Frugal innovation $\cdot$ vNOTES $\cdot$ gloveport

\section{Introduction}

Driven by the desire to minimalise surgical morbidity, the evolution from laparotomy to laparoscopy has now extended to the era of even less invasive surgery such as robotics, minilaparoscopy, single incision laparoscopic surgery (SILS) and natural orifice transluminal endoscopy (NOTES). Minimally invasive surgery not only improves cosmetic outcome, it also reduces the surgical injury, which in turn decreases the inflammatory and neuroendocrine response resulting in less post-operative pain and quicker recovery $[1,2]$.

NOTES attempts to reach the abdominal cavity through an invisible scar, i.e. numerous surgical procedures are performed via a natural body orifice. It has gained popularity amongst general surgeons, urologists and gastroenterologist over the past few years, and its feasibility and safety have been approved [3].

NOTES can be performed through a variety of approaches including the stomach, oesophagus, bladder and rectum, but the vast majority of NOTES procedures have been performed transvaginally, as the vagina provides direct access [4]. Culdotomy has been used widely for several surgical procedures (by gynaecologists but also by general surgeons for extraction of large specimens), and it has been approved as safe and easy to close $[5,6]$. 
In hybrid NOTES, the surgical procedure is performed through a natural body orifice with transabdominal assistance, whereas the term pure NOTES refers to procedures that involve only transluminal access.

Given its potential benefits, including no visible scars, fewer port-related complications and less painful and faster postoperative recovery, we introduced transvaginal pure NOTES (vNOTES) for benign adnexal masses in our surgical practice since November 2013. We describe here our initial clinical experience in poor man's tNOTES. We evaluate the feasibility and surgical outcome of vNOTES adnexectomy when performed with only conventional, reusable laparoscopic instruments, and an inexpensive, self-constructed single port device that can be quickly and easily assembled. We aimed to demonstrate that there is no need for expensive, commercially available disposable SILS-ports, other disposable instruments, or sealing devices, to perform a safe and equally time efficient adnexectomy by vNOTES.

\section{Materials and methods}

\section{Patients}

Between November 2013 and November 2014, a single surgeon (BJ) performed 20 adnexectomies by vNOTES. All patients were selected for adnexectomy for benign gynaecological disease. We selected each patient based on the following criteria: no contraindication for general anaesthesia, pneumoperitoneum or Trendelenburg position; no fixed uterus, strong pelvic adhesions or nodularity in the Pouch of Douglas on clinical examination; no history of pelvic inflammatory disease or moderate to severe endometriosis and mass not suspicious for malignancy. In our first 20 cases, we did not include patients with large fibroid uteri as these may impair visualisation. Virginity and pregnancy were considered as exclusion criteria whereas obesity $(\mathrm{BMI}=>>30)$ and absence of vaginal delivery were not.

The following patient and perioperative data were collected and retrospectively analysed: patient age, body mass index (BMI), parity, history of vaginal delivery, previous pelvic surgery, type of surgery, total operating time, serum haemoglobin $\mathrm{Hb})$ drop (change between the preoperative $\mathrm{Hb}$ and postoperative $\mathrm{Hb} 1$ day after surgery), (peri-) operative complications, post-operative pain score and size of the adnexal mass.

The duration of surgery was defined as the time from the start of colpotomy to the end of vaginal closure. Bowel, bladder, ureteral or vascular injuries, as well as blood loss $>300 \mathrm{ml}$, were considered as intraoperative complications. Short-term post-operative complications were classified as urinary tract infection, post-operative ileus, vaginal vault bleeding or infection or hematuria.
Post-operative pain was assessed using the visual analog pain scale (VAS) (scoring from $0=$ no pain to $10=$ worst imaginable pain). The VAS score was evaluated at 6 and $24 \mathrm{~h}$ post-operatively. All patients received the same intraoperative analgesia: intravenous paracetamol $1000 \mathrm{mg}$ and ketorolac trometamol $20 \mathrm{mg}$. Post-operative pain was managed by paracetamol $1000 \mathrm{mg}$, and ketorolac trometamol was administered on patient's demand.

No bowel preparation was done prior to surgery. A Foley catheter was placed just before surgery and removed the morning after surgery (range 12-22 h). Prophylactic intravenous antibiotic therapy, cefazoline $2 \mathrm{~g}$ and metronidazol $500 \mathrm{mg}$, was administrated during surgery.

As this was a new technique, the first patients were closely monitored post-operatively.

No vaginal intercourse was allowed for 6 weeks after the procedure. Each patient was re-assessed at the post-operative consultation 6 weeks after surgery.

\section{Surgical technique}

The patient was placed in lithotomy position under general anaesthesia. A rectovaginal examination was performed to exclude pelvic adhesions or obliteration of the Pouch of Douglas.

The operation field was disinfected and draped. A Foley catheter was placed. A $2.5-\mathrm{cm}$ single incision was made in the posterior vaginal fornix. The Pouch of Douglas was opened to insert the self-constructed NOTES port (Fig. 1). The device was constructed before surgery, using an Alexis Wound Protector/Retractor (Applied Medical, Rancho Santa Margarita, CA, USA) attached to a size 8 surgical glove. One finger of the surgical glove was incised to place a $10-\mathrm{mm}$ reusable trocar for $\mathrm{CO} 2$ insufflation and laparoscope insertion. Four 5$\mathrm{mm}$ reusable trocars were placed through the remaining fingers for insertion of the reusable laparoscopic instruments.

Assembling five trocars into the gloveport before starting the procedure gives the option of leaving instruments inserted through the trocars throughout surgery; thus, alternating between instruments becomes less time consuming. We used a

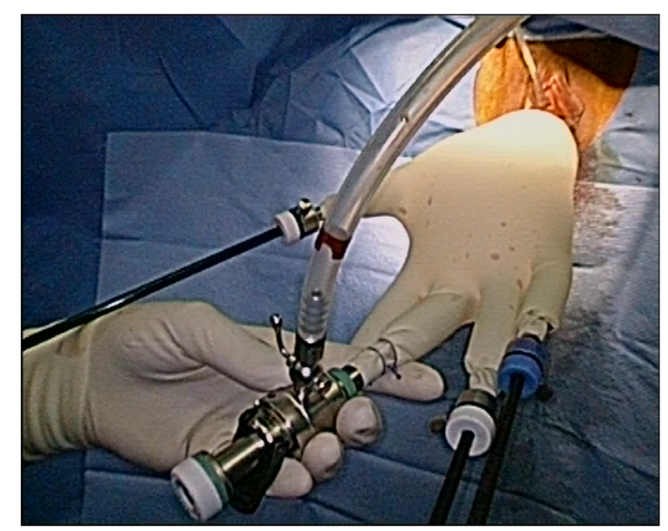

Fig. 1 Low cost self-constructed single port device 
standard rigid zero-degree 10-mm laparoscope. The reusable conventional laparoscopic instruments were a bipolar forceps, a pair of cold scissors, an atraumatic forceps, and a suctionirrigation cannula.

After placing the patient in Trendelenburg position, carbon dioxide was insufflated to maintain an adequate pneumoperitoneum.

Adnexectomy was performed (Video 1) by dissection of the infundibulopelvic and ovarian ligament, and fallopian tube, using the bipolar forceps and cold scissors. No sealing or ligature device was used.

After complete resection, the specimen was extracted through the wound protector into the glove part of the selfconstructed port. If the adnexal mass was too large, an endoscopic bag (Memobag $1200 \mathrm{ml}$; Teleflex) was used.The pursestring of the endobag was pulled through the colpotomy and was released outside of the vagina. Before removing the bag, the mass was first punctured to aspirate its content and reduce its volume. All adnexae were resected in toto, and no spilling into the abdominal cavity or vagina occurred.

After hemostasis and rinsing of the peritoneal cavity, the pneumoperitoneum was deflated and the port device removed with the specimen inside it. The colpotomy was closed using a Vicryl-1 (Ethicon, Piscataway, NJ, USA) running suture.

\section{Results}

Between November 2013 and November 2014, twenty adnexectomies were successfully performed by poor man's vNOTES using conventional, reusable laparoscopic instruments. No conversion to standard multi-incision laparoscopy or laparotomy was necessary. Fourteen patients underwent a unilateral adnexectomy. In six patients, a bilateral salpingooophorectomy was performed.

Table 1 presents an overview of patient and perioperative data. Individual patient details are presented in Table 2. Mean operation time was $32 \mathrm{~min}$. Five patients had had previous pelvic surgery. There were no intraoperative complications, and only one patient had a post-operative cystitis for which

Table 1 Overview of patient and perioperative characteristics

\begin{tabular}{lll}
\hline Data & Mean & Range \\
\hline Age (years) & 51 & $31-75$ \\
BMI $\left(\mathrm{kg} / \mathrm{m}^{2}\right)$ & 24.0 & $17.2-28.7$ \\
Total operating time (min) & 32 & $20-50$ \\
Serum hemoglobine drop (g/dl) & 0.9 & $0-2.1$ \\
Postoperative pain score & & \\
$\quad 6$ h & 2.0 & $0-4$ \\
$\quad 24 \mathrm{~h}$ & 1.3 & $0-2$ \\
Size of adnexal mass (mm) & 51.8 & $35-110$ \\
\hline
\end{tabular}

oral antibiotic therapy was administered. The mean drop in haemoglobin level was $0.9 \mathrm{~g} / \mathrm{dl}$. Most patients scored a low post-operative pain score (range $0-2$ ) $24 \mathrm{~h}$ after surgery. Mean size of the removed adnexal mass was $51.8 \mathrm{~mm}$ with the largest cyst measuring $110 \mathrm{~mm}$.

Each patient was examined 6 weeks after surgery. There was no vaginal wound infection nor dehiscence, and no patient complained of pain during pelvic examination. All patients were in good health and were all extremely satisfied with the result. One patient even mentioned playing tennis on day 1 post-surgery (against our medical advice).

\section{Discussion}

In this study, vNOTES for benign adnexal masses was successfully performed in 20 patients, using only conventional, reusable laparoscopic instruments and a self-constructed single incision port. The procedures were completed within a reasonable operation time and without complications. No conversion to laparotomy or standard laparoscopy was necessary.

An inexpensive, self-constructed single port device, which can quickly and easily be made by any surgeon, was used. Combining this self-constructed port device with easily available, conventional and reusable laparoscopic instruments, this study shows that adnexectomy via vNOTES is feasible without increasing the cost of laparoscopic surgery. This frugally innovative technique can potentially be performed in a low resource setting. It has been described as an approach for adhaesiolysis [7].

To the best of our knowledge, this is the first report of pure vNOTES for adnexectomy using this combination of a low cost port device with only reusable laparoscopic instruments.

A self-constructed port using a surgical glove has advantages when compared to commercial ports. It is less costly, it has flexible material that enables greater manipulation of instruments and a greater number and size of instruments can be passed through the incision.

Conventional transvaginal surgery has significant advantages compared to laparoscopic surgery such as the absence of abdominal scarring, reduced spillage by removing the specimen through the colpotomy and faster recovery from surgery [8]. By performing vNOTES, technical drawbacks of transvaginal surgery such as limited visualisation to attempt good hemostasis and the difficulty to perform adnexectomy in case of adhesions between the adnexa and the uterus can be overcome. Additionally, vNOTES abandons the risk of trocar related complications and induces less post-operative pain [9]. We did not encounter any difficulties in inserting the instruments using our poor man's vNOTES technique.

To perform vNOTES, various technical difficulties, comparable to those for transumbilical SILS, need to be overcome. The surgeon has to deal with problems due to instrument 
Table 2 Patient and perioperative characteristics of consecutive patients

\begin{tabular}{|c|c|c|c|c|c|c|c|c|c|c|c|c|}
\hline \multirow[t]{2}{*}{$\begin{array}{l}\text { Patient } \\
\text { no. }\end{array}$} & \multirow[t]{2}{*}{$\begin{array}{l}\text { Age } \\
\text { (years) }\end{array}$} & \multirow[t]{2}{*}{$\begin{array}{l}\text { BMI } \\
\left(\mathrm{kg} / \mathrm{m}^{2}\right)\end{array}$} & \multirow[t]{2}{*}{ Parity } & \multirow{2}{*}{$\begin{array}{l}\text { History of } \\
\text { vaginal } \\
\text { delivery }\end{array}$} & \multirow{2}{*}{$\begin{array}{l}\text { Previous } \\
\text { pelvic } \\
\text { surgery }\end{array}$} & \multirow[t]{2}{*}{$\begin{array}{l}\text { Type of } \\
\text { surgery }\end{array}$} & \multirow{2}{*}{$\begin{array}{l}\text { Total } \\
\text { operating } \\
\text { time (min) }\end{array}$} & \multirow{2}{*}{$\begin{array}{l}\text { Serum hemo- } \\
\text { globine drop } \\
(\mathrm{g} / \mathrm{dl})\end{array}$} & \multirow{2}{*}{$\begin{array}{l}\text { (Peri-) } \\
\text { operative } \\
\text { complications }\end{array}$} & \multicolumn{2}{|c|}{$\begin{array}{l}\text { Post-operative } \\
\text { pain score }\end{array}$} & \multirow{2}{*}{$\begin{array}{l}\text { Size of adnexa } \\
\text { mass (largest } \\
\text { diameter, mm) }\end{array}$} \\
\hline & & & & & & & & & & $6 \mathrm{~h}$ & $24 \mathrm{~h}$ & \\
\hline 1 & 54 & 24.1 & $\mathrm{P} 4$ & Yes & LS & $\mathrm{BSO}$ & 40 & 0.4 & - & 2 & 2 & 70 \\
\hline 2 & 44 & 17.2 & $\mathrm{P} 1$ & Yes & - & USO R & 35 & 0.8 & - & 2 & 2 & 62 \\
\hline 3 & 56 & 21.5 & $\mathrm{P} 2$ & Yes & LS & $\mathrm{BSO}$ & 35 & 0.5 & Cystitis & 2 & 2 & 35 \\
\hline 4 & 47 & 27.1 & $\mathrm{P} 2$ & Yes & - & USO R & 30 & 0 & - & 2 & 1 & 50 \\
\hline 5 & 58 & 26.0 & P0 & No & - & $\mathrm{BSO}$ & 35 & 0.6 & - & 4 & 1 & 40 \\
\hline 6 & 52 & 28.3 & P0 & No & - & USO R & 35 & 0.6 & - & 1 & 1 & 36 \\
\hline 7 & 66 & 22.9 & $\mathrm{P} 2$ & Yes & - & BSO & 40 & 0.7 & - & 2 & 1 & 45 \\
\hline 8 & 46 & 20.8 & P0 & No & - & USO R & 22 & 1.4 & - & 2 & 1 & 35 \\
\hline 9 & 51 & 25.4 & $\mathrm{P} 2$ & Yes & - & USO L & 22 & 0.5 & - & 2 & 1 & 35 \\
\hline 10 & 56 & 24.2 & $\mathrm{P} 1$ & Yes & - & USO R & 25 & 1.2 & - & 2 & 1 & 42 \\
\hline 11 & 63 & 26.7 & $\mathrm{P} 2$ & Yes & - & $\mathrm{BSO}$ & 30 & 2.0 & - & 3 & 0 & 40 \\
\hline 12 & 56 & 25.0 & $\mathrm{P} 2$ & Yes & - & USO R & 22 & 0.5 & - & 1 & 1 & 39 \\
\hline 13 & 75 & 23.2 & $\mathrm{P} 1$ & Yes & - & USO R & 20 & 0.6 & - & 2 & 2 & 38 \\
\hline 14 & 31 & 21.5 & $\mathrm{P} 2$ & Yes & - & USO R & 35 & 1.8 & - & 2 & 2 & 60 \\
\hline 15 & 45 & 28.7 & P1 & Yes & - & USO R & 20 & 0 & - & 2 & 2 & 40 \\
\hline 16 & 43 & 24.4 & P2 & No & $\mathrm{CS}$ & USO R & 50 & 0.9 & - & 2 & 2 & 100 \\
\hline 17 & 45 & 23.7 & P2 & Yes & $\mathrm{CE}$ & USO R & 45 & 0.7 & - & 0 & 0 & 110 \\
\hline 18 & 36 & 22.8 & $\mathrm{P} 2$ & Yes & $\mathrm{CS}$ & USO R & 40 & 1.7 & - & 2 & 1 & 39 \\
\hline 19 & 55 & 23.4 & P1 & Yes & - & BSO & 35 & 1.2 & - & 2 & 1 & 70 \\
\hline 20 & 38 & 22.5 & $\mathrm{P} 2$ & Yes & - & USO L & 32 & 2.1 & - & 2 & 2 & 49 \\
\hline
\end{tabular}

$C E$ cystectomy, $C S$ caesarean section, $L S$ laparoscopic sterilisation, USO unilateral salpingo-oophorectomy, BSO bilateral salpingo-oophorectomy, $R$ right, $L$ left

collision, limited triangulation and reduced traction of tissue $[10,11]$. Due to the colpotomy providing a more flexible entry compared to the infraumbilical fascia opening, we find these difficulties to be less restricting when compared with SILS.

As the camera is inserted through the Pouch of Douglas, the view through a vNOTES port is opposite to the standard laparoscopic view, i.e. caudal to cranial as opposed to cranial to caudal. Rotating the axis of the surgical field for vNOTES required a brief adaptation period.

For the poor man's vNOTES technique, a standard rigid $0^{\circ}$ endoscope was used and provided good visualisation. Experience gained from performing vNOTES adnexectomies in a non poor man's setting, showed that a $30^{\circ} 5-\mathrm{mm}$ chip on tip endoscope provides even better visualisation and maneuverability.

One could argue the possibility of pelvic infection after vaginal surgery; however, none of our patients presented with this complication after the vNOTES procedure. Previous studies have also shown that post-operative pelvic infection is unlikely to happen especially when prophylactic antibiotics are administered [12, 13]. Further concern when performing transvaginal surgery is the development of post-operative dyspareunia. No difference between conventional compared to laparoscopic transvaginal surgery is to be expected, and different studies show the absence of dyspareunia at a midand long-term follow-up [12-14]. As was the case for our study protocol, sexual abstinence should be recommended for 6 to 8 weeks as is the recommendation for conventional transvaginal surgery [14].

Some contra-indications should be considered before performing vNOTES. In patients with a massive hemoperitoneum, the endoscopic view will get disturbed [15]. If Pouch of Douglas adhesions can be expected, a thorough pelvic examination should be performed prior to surgery, and in case of unexpected Pouch of Douglas obliteration, conversion to transabdominal laparoscopy should be considered. Virginity is another contra-indication for vNOTES. On the other hand, nulliparity nor absence of history of vaginal delivery should be a reason not to perform vNOTES. In our case series, the highest BMI was 28.7. We did not, however, consider obesity $(\mathrm{BMI}=>>30)$ a contra-indication. If a good Trendelenburg position can be achieved, the bowel and mesentary can be lifted out of the pelvis and will not impair visualisation.

Lee et al. [15] suggested that vNOTES should be avoided in patients with an adnexal mass larger than $80 \mathrm{~mm}$ due to technical difficulties. We, however, successfully managed to perform adnexectomy by vNOTES in two patients with an 
adnexal mass larger than $80 \mathrm{~mm}(110$ and $100 \mathrm{~mm}$, respectively) without significantly increasing the duration of surgery. All adnexae were resected in toto. In case of a large mass, an endoscopic bag was used. The pursestring of the endobag was pulled through the colpotomy and released outside the vagina. Before removing the bag, the mass was first punctured to aspirate its content and reduce its volume. All adnexae were resected in toto, and no spilling into the abdominal cavity or vagina occurred.

As previously mentioned by Lee et al. [15], the major limitation of vNOTES is the inability to overview the pelvicarea, in particular the vesico-uterine pouch, and thus lesions such as bladder endometriosis or anterior uterine wall myomas can be missed. Innovation of endoscopes is desirable to overcome this limitation and to have the ability with vNOTES to explore the entire abdominal cavity.

Further concern is the possibility of performing repeated vNOTES procedures. To date, only one group of surgeons has reported on repeated vNOTES, performed in two patients 6 and 8 months after previous vNOTES [16].

\section{Conclusion}

In this study, poor man's vNOTES for benign adnexal masses was successfully and safely completed in correctly selected patients. We showed this frugally innovative technique to be feasible with only reusable standard laparoscopic instruments and a low cost self-constructed single port device. This technique may potentially be applied in a low resource setting. Adnexal masses up to $110 \mathrm{~mm}$ could be easily and safely removed without spillage of contents into the abdomen. Less post-operative pain and a quicker recovery are recorded as other advantages of vNOTES. Additionally, it avoids abdominal wall wounds and trocar-related complications. The major limitation of vNOTES with the currently available instruments and endoscopes appears to be the inability to explore the vesico-uterine pouch. Innovations in instruments and endoscopes together with additional studies with larger cohorts and longer follow-up should lead to vNOTES being used to safely perform more complex gynaecological procedures in most patients suitable for laparoscopic surgery.

Conflict of interest Anneleen Reynders and Jan Baekelandt declare that they have no conflict of interest.

Contributions Anneleen Reynders collected the data, performed the literature review and wrote the article. She was first assistant during most of the procedures.

Jan Baekelandt recruited and operated all the patients. He supervised the data collection, reviewed and submitted the article and made the video.
Ethics All procedures followed were in accordance with the ethical standards and with the Helsinki Declaration of 1975, as revised in 2008 [5]. Informed consent was obtained from all patients for being included in the study.

\section{References}

1. Burpee SE, Kurian M, Murakame Y, Benevides S, Gagner M (2002) The metabolic and immune response to laparoscopic versusopen liver resection. Surg Endosc 16(6):899-904

2. Grande M, Tucci GF, Adorisio O et al (2002) Systemic acutephaseresponse after laparoscopic and open cholecystectomy. Surg Endosc 16(2):313-316

3. Rattner D, Kalloo A (2006) ASGE/SAGES working group on natural Orifice translumenal endoscopic surgery. October 2005. Surg Endosc 20:329-333

4. Santos BF, Hungness ES (2011) Natural orifice translumenal endoscopic surgery:progress in humans since white paper. World J Gastroenterol 17:1655-1665

5. Tolcher MC, Kalogera E, Hopkins MR, Weaver AL, Bingener J, Dowdy SC (2012) Safety of culdotomy as a surgical approach: implications for natural orifice transluminal. JSLS 16:413-420

6. Uccella S, Cromi A, Bogani G, Casarin J, Serati M, Ghezzi F (2013) Transvaginal specimen extraction at laparoscopy without concomitant hysterectomy: our experience and systematic review of the literature. J Minim Invasive Gynecol 20:583-590

7. Baekelandt J (2014) Poor Man's NOTES: can it be a good approach for adhesiolysis? a first case report with video demonstration. J Minim Invasive Gynecol 4650(14):01530-01531. doi:10.1016/j. jmig.2014.11.001

8. Ferrari MM, Mezzopane R, Bulfoni A et al (2003) Surgical treatmentof ovarian dermoid cysts: a comparison between laparoscopicand vaginal removal. Eur J Obstet Gynecol Reprod Biol 109:88-91

9. Hackethal A, Sucke J, Oehmke F et al (2010) Establishing transvaginalNOTES for gynecological and surgical indications: benefits, limits, and patient experience. Endoscopy 42:875-878

10. Peak J, Kim SW, Lee SH et al (2011) Learning curve and surgical outcome for single-port access total laparoscopic hysterectomy in 100 conscutive cases. Gynecol Obstet Investig 72:227-233

11. Phongnarisorn C, Chinthakanan O (2011) Transumbilical singleincision laparoscopic hysterectomy with conventional laparosopic instruments in patients with symptomatic leimyoma and/or adenomysosis. Arch Gynecol Obstet 284:893-900

12. Zornig C, Mofid H, Siemssen L et al (2009) Transvaginal NOTES hybrid cholecystectomy:feasibility results in 68 cases with midterm follow-up. Endoscopy 41:391-394

13. Lee CL, Wu KY, Su H, Wu PJ, Han CM, Yen CF (2014) Hysterectomy byTransvaginal Natural Orifice Transluminal Endoscopic Surgery (NOTES): a series of 137 patients. JMIG 21(5):818-824

14. Tanaka M, Sagawa T, Yamazaki R, Myojo S, Dohi S, Inoue M (2013) Evaluation of transvaginal peritoneal surgery in young femalepatients. Surg Endosc 27:2619-2624

15. Lee CL, Wu KY, Su H, Ueng SH, Yen CH (2012) Transvaginal Natural-Orifice Transluminal Endoscopic Surgery(NOTES) in Adnexal Procedures. JMIG 19:509-513

16. Perretta S, Vix M, Dallemagne B, Nassif J, Donatelli G, Marescauw J (2012) Repeated transvaginal notes: is it possible? Surg Endosc 26:565 